\title{
Hierarchical Control Architecture of Co-located Hybrid Power Plants
}

This paper was downloaded from TechRxiv (https://www.techrxiv.org).

\section{LICENSE}

CC BY 4.0

SUBMISSION DATE / POSTED DATE

05-09-2021 / 10-09-2021

CITATION

Long, Qian; Das, Kaushik; Sørensen, Poul Ejnar (2021): Hierarchical Control Architecture of Co-located Hybrid Power Plants. TechRxiv. Preprint. https://doi.org/10.36227/techrxiv.16571094.v1

$\mathrm{DOI}$

10.36227/techrxiv.16571094.v1 


\title{
Hierarchical Control Architecture of Co-located Hybrid Power Plants
}

\author{
Qian Long, Member, IEEE, Kaushik Das, Senior Member, IEEE, and Poul Sørensen, Fellow, IEEE
}

\begin{abstract}
The utility-scale co-located hybrid power plants (HPPs) have been receiving attention globally due to enhanced controllability and efficient utilization of electrical infrastructure. While power plant control has been extensively studied for singletechnology power plants in the past decades, how to control a colocated HPP that includes sub-plants with multiple technologies is yet to be well defined. To fill the gap, this paper proposes a novel hierarchical control architecture for co-located HPPs. This control architecture contains four control levels: asset control level, plant control level, HPP control level and HPP energy management system (EMS) level. The objective of HPP EMS level is to find optimal strategies for market participation, and the objective of HPP control level is to execute those strategies from the HPP EMS in real time. The interactions across the control hierarchy are firstly discussed in this paper, where attention is closely paid to interactions between HPP EMS level and HPP control level, and between HPP control level and plant control level. Novel strategies for control coordination are presented to ensure all the control levels work together without counteracting against each other. Frequency control and fault ride-through are two examples to demonstrate such control coordination.
\end{abstract}

Index Terms-hierarchical control architecture, hybrid power plants, asset control level, plant control level, control coordination.

\section{INTRODUCTION}

\section{A. Background}

$\mathbf{T}$ HE development of renewable energy sources (RES) technologies, such as wind and solar, has progressed significantly due to technology improvements, cost reduction and policy support [1]. Competitive RES costs and continued policy support suggest that the shares of RES will continue to increase globally. However, due to variability and uncertainty associated with RES, new strategies that increase grid operation flexibility are called for. Energy storage (ES), as one strategy for facilitating high shares of RES, has been increasingly integrated into the grid. There has been recently an interest in combining RES with ES technology to form utility-scale co-located hybrid power plants (HPPs). According to the study [1], the main advantages of the co-located HPPs are potential increased market value benefits due to enhanced controllability, and reduced project costs thanks to efficient utilization of electrical infrastructure. A number of conducted research studies and the first built HPPs have demonstrated the expanded capabilities of HPPs [2].

In order to realize enhanced controllability of HPPs, proper energy management systems (EMSs) and control systems are necessary to maximize the revenue while to guarantee that the operation complies with the grid requirements. Before the design of EMS and control systems comes into place, a control architecture design is in need. However, to date, only a few works concern control architecture of HPPs that supports one specific control function and no existing studies discuss a generic control architecture design for HPPs. To fill the gap, this paper proposes a generic hierarchical control architecture for HPPs that takes into account different control levels and control functions.

Hierarchical control has been well known for the applications in power systems, such as power dispatch and voltage control [3], [4]. It has also been used extensively for decades in renewable power plants like wind power plants (WPPs) and solar power plants (SPPs). With the rise of powerelectronics-based microgrids, hierarchical controls have also been successfully applied to manage both grid-connected and islanded operation of microgrids [5]. In the next subsection, a literature review is presented regarding the application of hierarchical control in utility-scale renewable power plants, which is the focus of this paper.

\section{B. Literature Review}

While control of inverter-based grid-connected RES has been well-established [6], how to manage single-technology power plants (i.e. WPPs, SPPs and ESSs) in terms of active/reactive power control and grid supporting functions has been still an active area in both research activities and demonstration projects [7]-[11]. Hierarchical control architecture has been mostly adopted in single-technology renewable power plants. This architecture involves two control levels, that is, a plant control level that manages power flows at the plant point of connection ( $\mathrm{PoC}$ ) and dispatches control commands to individual assets, and an asset control level that addresses local control function at each unit.

Active power control enables renewable power plants to inject active power to the grids. The plant controller allocates the total active power reference to each asset based on its available power, and the asset controller tracks its own active power reference [12]. For dispatchable resources like ES, it is straightforward to regulate active power output using power electronic devices, whereas extra control functions are required for non-dispatchable resources like wind and solar power to enable flexible regulation of active power, including derated operation [13], [14], DC-link voltage control [15], and rotor speed or pitch control (only for WTs) [16], [17].

Given active power capabilities at asset control level, the frequency controller can be implemented at either plant control level or asset control level. Authors from [12] and [18] discuss the auxiliary loop for frequency control at plant control level 
for WPPs. Proportional-integral (PI) controllers are adopted for SPP control to provide fast frequency response [19]. Authors from [20] propose a linear-quadratic-regulator-based frequency controller for SPPs to track a desired frequency response with designed inertia and droop coefficients. However, considering communication delays and control design requirements, the time response of plant controllers can be slow and fail to meet the technical requirements of fast frequency reserve (FFR). Therefore, fast frequency controllers have been mostly implemented at asset control level [16], [21]-[23]. However, during the provision of frequency services, plant control level will counteract against asset control level, if no coordination is considered. This counteraction issue arises when there is immediate power injection triggered at asset control level and captured as control feedback at plant PoC. It results in undesired control actions at plant control level which suppress frequency response triggered at asset control level. So far, few work has been done to address the coordination between plant control level and asset control level for frequency response.

Reactive power control has been implemented to enable voltage support and FRT capabilities of renewable power plants. The plant controller regulates reactive power, voltage or power factor depending on the utility's interconnection requirement or control modes, and dispatches reactive power or delta voltage reference to the asset controllers. The hierarchical voltage control architecture is described for WPPs [18]. In [24], a centralized WPP voltage control is proposed with reactive power capability calculation based on detailed WPP power collection system (PCS) model, wake effects of WPP, and converter voltage and current limits. Although FRT functions are performed at asset control level [25], existing studies show that the plant controller contributes to better short-term voltage stability [26], [27]. In [27], the plant control level is proposed for SPPs to maintain voltage at the plant $\mathrm{PoC}$ at the upper voltage limit to avoid overvoltage during the fault recovery stage. An adaptive hierarchical voltage control for WPPs is presented in [28], where the plant controller dispatches delta voltage reference to each WT and utilizes the washout filter to prevent the overvoltage after the fault clearance. However, there is few work that focuses on how to coordinate different control levels such that counteraction is avoided during FRT period.

There are currently a few literature on co-located HPP control. Power smoothing control is proposed in [29] to reduce the output power fluctuations of wind and solar and to manage battery state of charge. In [30], a solar-storage HPP controller for active power control has been presented, where ramp rate limitations and frequency control are integrated to fulfill grid code requirements. Authors in [31] implement a control strategy for a HPP consisting of a WPP, a SPP and an ESS, and the study pays special attention to a coordinated frequency response of the HPP. However, all the above-mentioned papers that consider frequency responses implement frequency control schemes at HPP control level. This design could limit the types of frequency services a HPP could provide. For example, the communication delay from HPP control to asset control could undermine the capability of HPP for providing fast frequency response, which requires a short activation time. Besides, none of the papers have provided a detailed view on the interactions between different control levels.

\section{Contribution and Outline}

In this paper, a novel hierarchical control architecture for utility-scale co-located HPPs is presented. The main contribution of this paper is summarized as follows:

1) the control hierarchy, along with the role of each level, is defined in detail in terms of how to manage sub-plants with multiple technologies and enable HPP control functions. Variables exchanged between control levels are also specified in order to better understand interactions across control hierarchy;

2) two new control levels are introduced, namely, HPP EMS level and HPP control level, with a novel HPP controller design being proposed;

3) a coordination strategy is developed to ensure all the control levels support each other without counteraction, especially during the provision of time-critical functions, such as frequency control and fault ride-through (FRT);

4) priorities among all the control functions are taken into account in order to allow stacking operation of multiple control functions.

Next section introduces the proposed control architecture and outlines each control level. Control functions, including active power control, frequency control, reactive power and voltage control, and FRT, are described in Section III, where the focus is on interactions between different control levels. Section IV concludes the paper.

\section{Hierarchical Control Architecture}

\section{A. Hierarchy}

Fig. 1 shows the system structure of a generic co-located HPP. Individual wind turbine (WT) units, photovoltaic (PV) units and ES units are aggregated into a WPP, a SPP and an ESS, respectively, via each of its own PCS. The sub-plants are aggregated as the HPP via the HPP PCS. Each WT, PV or ES unit has measurement points at its terminal (shown as $w t_{i}, p v_{i}, e s_{i}$ and $s t_{i}$ in Fig. 1) to provide information for asset controllers. Measurement points are also located at the PoC of each sub-plant (shown as wpp, spp, ess and aux in Fig. 1) to make measurements available for plant controllers and so are the ones at the HPP PoC (shown as hpp in Fig. 1).

A hierarchical control architecture is proposed in Fig. 2, with a total of four control levels. From top to bottom, there is the HPP EMS level, the HPP control level, the plant control level and the asset control level. In Fig. 2, the higher control level sends control commands and parameters to its lower control level, shown as red arrows, and the lower control level sends back system states and measurements to its higher control level, shown as blue arrows. These four control levels are described in the following subsection, and interactions between two neighboring control levels are discussed in Section III.

Time-scale separation must be taken into account when such control architecture is designed. The main principle of defining 


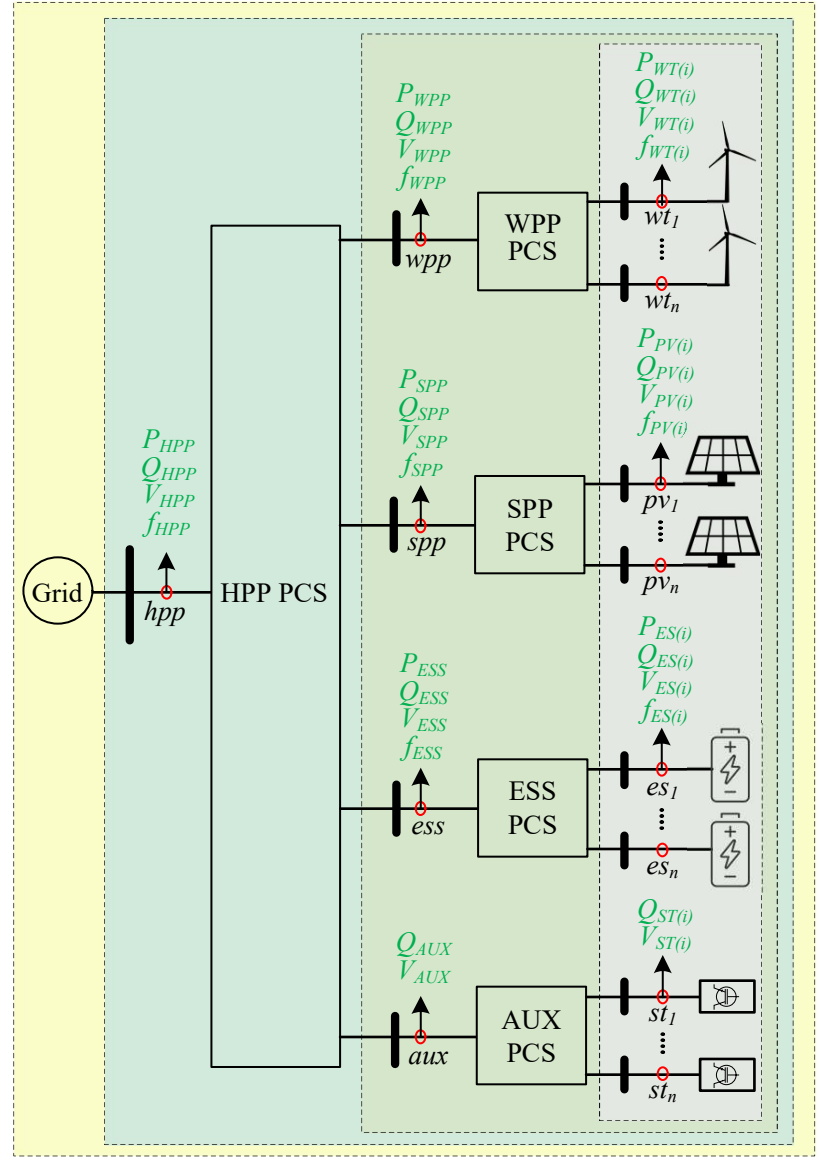

Fig. 1: Structure of HPP PCS with measurement points.

the time resolution of each control level is that, a higher control level should have a larger time resolution and vice versa. The asset control level acts in the range of milliseconds to seconds, dictated by power converter control. Both plant control level and HPP control level act in the range of seconds to minutes. However, the HPP controller needs to be adjusted to a slower response than the plant controllers in order to avoid undesired control counteractions between the two. Finally, HPP EMS level acts in minutes up to hours range.

\section{B. HPP EMS}

The objective of the HPP EMS is to maximize the revenue stream of the HPP while to consider utility regulations and physical limits of the power plants. This control level optimizes the revenue by deciding the energy sold in the energy and balancing markets and the reserve secured for the ancillary service market based on market price forecast and renewable forecast.

The HPPs need to bid in the reserve markets, day-ahead spot market, intra-day market and balancing market based on different forecasts at different time horizons. Generally, the power reserves are bid in the reserve market either with long-term contract or purchased by the system operators in short term but before the spot market opens, typically two days before the real-time. Spot market is where bulk transactions of power takes place generally one day before

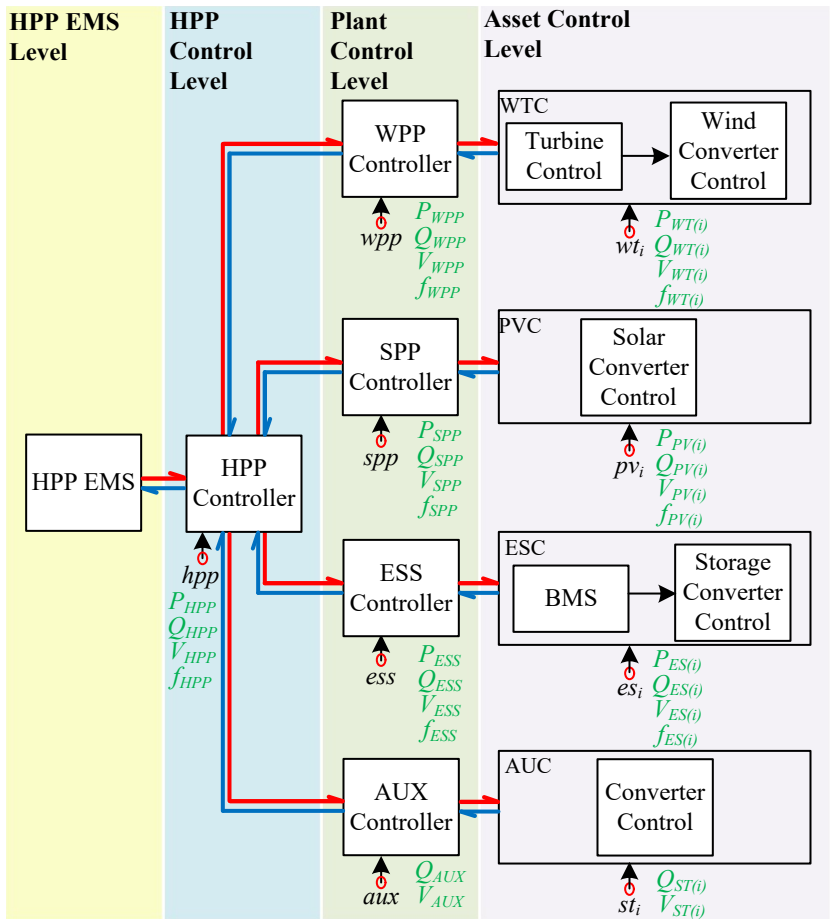

Fig. 2: HPP hierarchical control architecture.

the real-time. Following the day-ahead spot market, further bids can be performed by the power plant operators in intraday (typically from one day to one hour before the real-time) and/or balancing market (typically one hour before the realtime). In the balancing market, the power plant operators have the privilege of improved forecast; therefore, either to mitigate the penalties due to forecast error in the spot market bidding or to earn revenue from the balancing market by mitigating imbalances from other sources of the system.

\section{HPP Control}

There are five main tasks that the HPP controller should fulfill:

1) ensures that the sold power reserve is available at any instant. This task is relevant to the HPP frequency control functionality and the value stream the HPP can obtain from the reserve market.

2) ensures that the sold energy is provided within a specified time window in a way that ramp rate limitations are respected. This task is relevant to the HPP active power control functionality and the value stream the HPP can obtain from spot and balancing markets.

3) ensures that an absolute upper power limit is kept in order to limit HPP power injection at any instant. This task guarantees that the grid constraints are respected. It also complements the second task in terms of active power control functionality.

4) ensures that reactive power output at the HPP PoC follows the requirements from the power system operators. This task addresses the responsibility of the HPP controller for regulating reactive power at the HPP PoC. 
5) ensures that voltage at the HPP PoC follows the requirements from power system operators. This task addresses the responsibility of the HPP controller for regulating voltage at the HPP PoC.

With the consideration of the above tasks, the HPP active power control and reactive power control are proposed in Fig. 3 . The energy reference from the HPP EMS $E_{H P P}^{*}$ refers to the energy that the HPP is supposed to provide within a specified time window, which is determined by the highest EMS time resolution. The HPP active power control takes $E_{H P P}^{*}$ as control reference. The mismatch between energy reference and energy output is divided by the remaining time in the time window $T_{\text {rem }}$, yielding the HPP active power reference $P_{H P P}^{*}$. The HPP active power reference $P_{H P P}^{*}$ is subject to a saturation limiter and a ramp rate limiter. An antiwindup PI controller is then used to track the HPP active power output, and to avoid error accumulation due to any input error. The PI controller output is limited by $P_{H P P N}$, the total capacity of the operational units in the HPP. Eventually, the saturated active power reference $P_{\sum}^{*}$ acts as the input for the HPP dispatch function. As for the HPP reactive power control, it has two modes: reactive power tracking and voltage tracking. The HPP reactive power setpoint and the HPP PoC voltage setpoint are defined by grid connection requirements and sent by system operators. Similar to active power control, the difference between the setpoint and the measured output is regulated by an anti-windup PI controller, whose output acts as the input to the HPP dispatch function. The HPP dispatch function decides active and reactive power reference, along with the reserve reference for plant control level, based on the HPP controller outputs and the information received from HPP EMS level and plant control level. The implementation details of the HPP dispatch function is discussed in Section III.

\section{Plant Control}

Plant control level ensures that references from HPP control level are met at the plant PoC. The two major control loops in the plant controllers are the active power control loop and the reactive power control loop. These two control loops receive power references from HPP control level, and through PI controllers generate power references for asset control level. On top of these two control loops, auxiliary control loops can be added to achieve advanced control applications, which consist of: 1) active power control, including balance control, delta control and ramp rate control [12], 2) frequency control, 3) reactive power control, 4) voltage control, and 5) FRT coordination [18]. Other than the control applications above, control applications such as state of charge management and oscillation damping control may also be considered at plant control level for ESSs [32].

A dispatch block is included in each power plant controller to generate power references for all the assets within the power plant. The dispatch block can be implemented in multiple ways to best utilize the infrastructure. Among those, three types of dispatch algorithms are described as follows: 1) Dispatch Based on Availability, where the power reference for each unit is generated based on the level of available power each unit holds; 2) Dispatch Based on Rating: the power reference for each unit is generated based on the rated power of each unit; 3) Optimization: active and reactive power dispatch are formulated as a constrained optimization problem generating optimal active power and reactive power for each asset. The optimization objective includes minimizing PCS losses within the plant, maximizing the utilization of available power, etc.

\section{E. Asset Control}

The asset controllers guarantee that output voltage and current at the asset terminals are regulated based on control commands they receive from plant control level. As seen in Fig. 2, a general diagram of the asset controller of a WT, a PV and an ES is described. The WT asset controller consists of turbine control and wind converter control. The turbine control represents the electro-mechanical control system in the WT, including blade pitch control, pitch compensation and torque control. The wind converter control includes active power control and reactive power control that dictate the power to be delivered to the system [14]. The bulk of the PV asset controller is solar converter control, which includes maximum power point tracking (MPPT) control, active power control, and reactive power control. The ES asset controller is made up of the battery management system (BMS) and storage converter control. The BMS provides monitoring and protection functions for battery pack and controls the charging and discharging profile, while the storage converter control determines the bidirectional power flow interacted with the grid, based on references from plant control level and status information provided by the BMS. To become subject to the grid codes, additional frequency control can be added as outer control loops to each asset controller mentioned above, such as droop-based frequency regulation and synthetic inertia, and likewise voltage control, such as droop-based voltage regulation, fixed reactive power control and power factor control.

\section{HPP CONTROL FUNCTIONS}

The control functions being considered within the scope of this paper include active power control, frequency control, reactive power control, voltage control and FRT.

\section{A. Interactions Between Control Levels}

This subsection discusses the variables exchanged in the control hierarchy. These variables enable control functions that are discussed in the rest of the section. Fig. 4 shows the variables exchanged between HPP EMS level and HPP control level as well as the ones between HPP control level and plant control level. The variables exchanged between plant control level and asset control level are usually well-defined and customized by manufacturers. Therefore, those variables are not described in this paper, but accommodated by the proposed control architecture.

What the HPP EMS sends to the HPP controller are mainly energy and active power references that are generated by EMS 


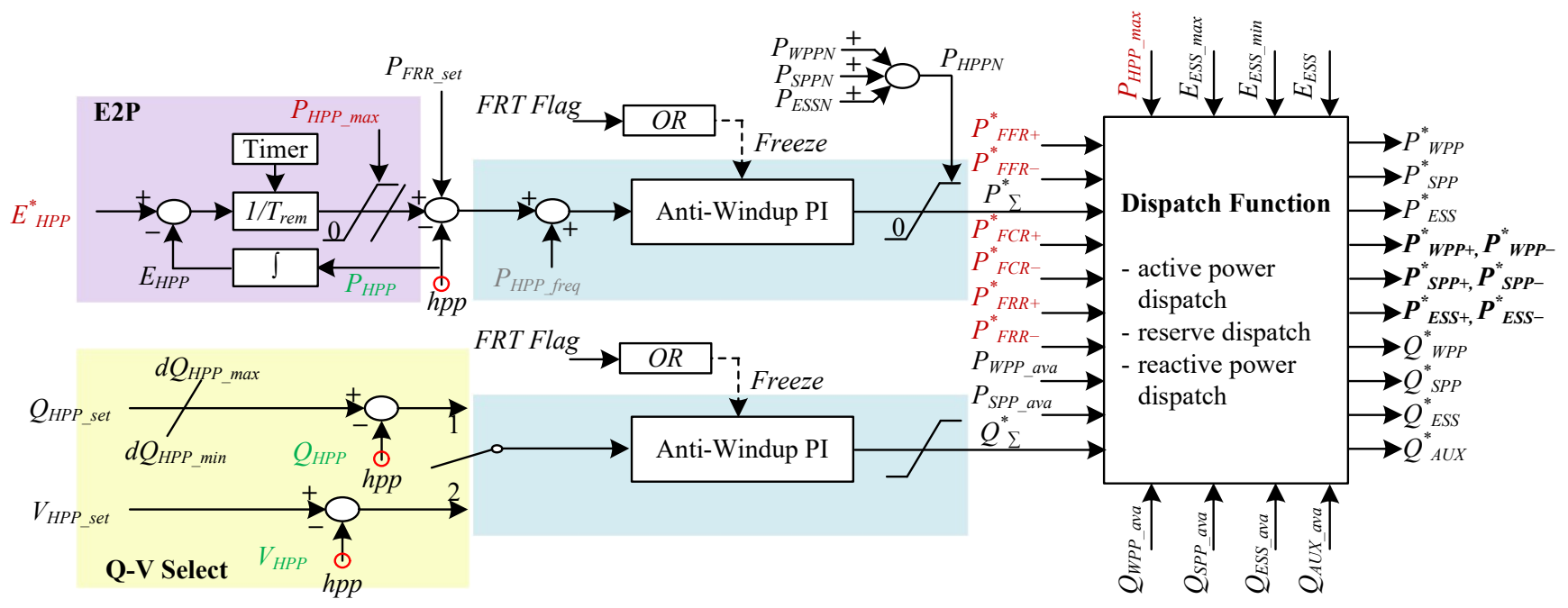

Fig. 3: HPP controller design.

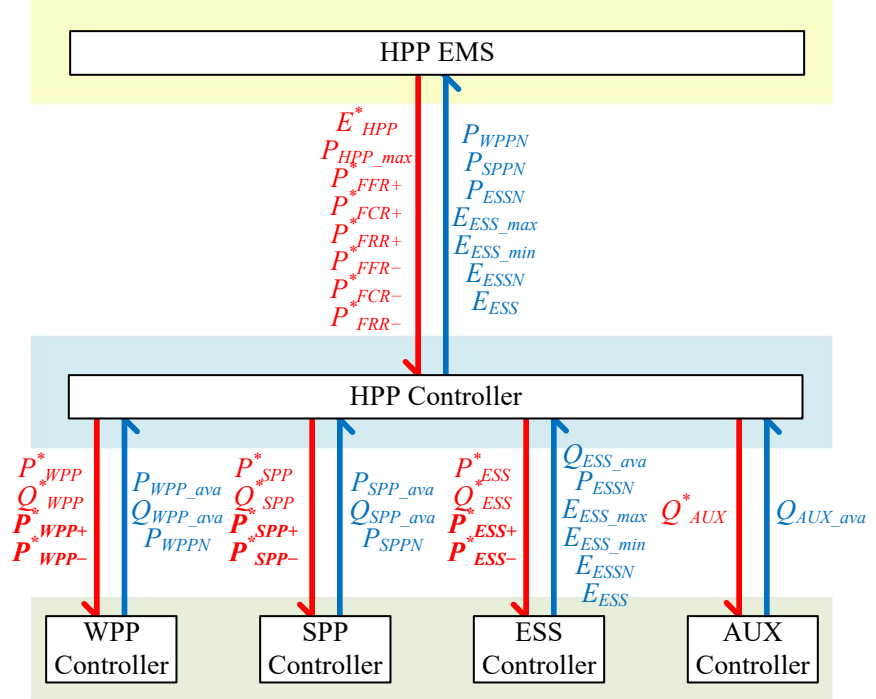

Fig. 4: Variables exchanged across the control hierarchy.

optimization algorithms. $E_{H P P}^{*}$ is updated according to the energy bids of the HPP in the energy market. The absolute maximum power limit $P_{H P P_{-} \max }$ behaves as an indicator of the operating priority between ESS charging and RES curtailment. If $P_{H P P_{-} \max }$ is large, it indicates that a high HPP power output is favorable and so is the MPPT mode with the WPP and the SPP. If $P_{H P P \_\max }$ turns out to be small, it implies that the WPP and the SPP might need to initiate power curtailment to make sure the HPP power output doesn't exceed $P_{H P P_{-} \max }$. Upward and downward reserve references correspond to the amount of the reserves the HPP has committed in the reserve market. It is assumed that there is mostly no operation conflict between energy references and reserve references, thanks to the optimization from the HPP EMS. However, when the conflict arises, several priorities have to be followed by the HPP controller: 1) securing reserves has higher priority than tracking energy references, 2) FFR has the highest priority, followed by frequency containment reserve (FCR) and frequency restoration reserve (FRR), 3) securing upward reserves has higher priority than downward reserves, 4) tracking $E_{H P P}^{*}$ has higher priority than tracking $Q_{H P P_{-} \text {set }}$ and $V_{H P P_{-} s e t}$ except for FRT.

The variables sent from the HPP controller to the HPP EMS are used as the update to make sure the HPP EMS optimizes the portfolio of energy bids and reserve bids using the latest relevant information from the HPP. $P_{W P P N}, P_{S P P N}$ and $P_{E S S N}$ reflect the power capacity of the WPP, the SPP and the ESS, respectively, considering all the online units. They equal to the nominal capacity when all the units are online, but are updated if any loss of the unit happens within the plant. The nominal energy capacity of the ESS $E_{E S S N}$ is updated likewise. A constraint is imposed on the ESS-related variables as follows:

$$
0<E_{E S S \_m i n} \leq E_{E S S} \leq E_{E S S \_m a x}<E_{E S S N}
$$

Compared to a slow rate at which the variables between the HPP EMS and the HPP controller are exchanged, the variables between the HPP controller and the plant controllers need to be updated faster for real-time control and dispatch. Among the variables sent from the HPP controller, active and reactive power references are sent to each plant controller (only reactive power reference for auxiliary plants). Two active power reserve vectors, $\mathbf{P}^{*} \mathbf{p}+$ and $\mathbf{P}^{*} \mathbf{p}-$, are also sent to the plant controllers for WPP, SPP and ESS. These two vectors contain the reserve reference for each type of frequency service, namely FFR, FCR and FRR. The variables the plant controllers send to the HPP controller include $P_{W P P N}, P_{S P P N}, P_{E S S N}$ and $E_{E S S N}$, which are updated in the same manner as mentioned earlier. Besides, $P_{p_{-} a v a}$ and $Q_{p_{-} a v a}$ are sent to the HPP controller as the input for active power reserve dispatch and reactive power dispatch, respectively. $E_{E S S}, E_{E S S_{-} \text {min }}$ and $E_{E S S_{-} \max }$ are used for active power dispatch. These dispatch algorithms are discussed in the following sections in detail. Several priorities have to be followed by the plant controllers: 1) securing reserves $\mathbf{P}^{*} \mathbf{p}+$ 
and $\mathbf{P}^{*} \mathbf{p}-$ has higher priority than tracking active power reference $\left.P_{p}^{*}, 2\right)$ FFR has the highest priority, followed by FCR and FRR, 3) securing upward reserves has higher priority than downward reserves, 4) tracking active power reference $P_{p}^{*}$ has higher priority than tracking reactive power reference $Q_{p}^{*}$ except during FRT periods.

\section{B. Active Power Control}

Under active power control, the main goal of the HPP controller is to make sure the HPP energy output tracks energy reference $E_{H P P}^{*}$ sent from the HPP EMS every EMS time step while to send real-time active power reference to each power plant, as shown in Fig. 3. The E2P block converts energy reference to active power reference which is sent to the antiwindup PI controller. The PI controller output is sent as the input to the HPP dispatch function which makes decision on dispatching active power to each power plant.

The control logic of the HPP dispatch function under active power control is shown in Fig. 5. The control logic starts with checking if the ESS available energy $E_{E S S}$ is within the lower and the upper energy limit $E_{E S S_{-} \text {min }}$ and $E_{E S S_{-} \text {max }}$, which represent the technical energy limit of the ESS, sent by the ESS controller. The maximum discharging and charging power, $P_{E S S \_m a x}$ and $P_{E S S \_m i n}$, are then updated accordingly. Whether the measured output power at HPP PoC $P_{H P P}$ is higher or lower than $P_{H P P_{-} \max }$ decides if the HPP enters State 1 (MPPT Operation) or State 2 (Power Curtailment). The active power dispatch references in State 1 are defined as below:

$$
\begin{gathered}
P_{W P P}^{*}=P_{W P P N} \\
P_{S P P}^{*}=P_{S P P N}
\end{gathered}
$$

$$
\begin{aligned}
& \text { if } P_{H P P_{-} \max } \geq P_{\sum}^{*}: \\
& P_{E S S}^{*}= \begin{cases}P_{E S S \_m i n} & P_{E S S}^{*} \leq P_{E S S \_m i n} \\
P_{E S S \_m a x} & P_{E S S}^{*} \geq P_{E S S \_m a x} \\
P_{\sum}^{*}-P_{W P P}^{*}-P_{S P P}^{*} & o . w .\end{cases} \\
& \text { if } P_{H P P \_\max }<P_{\sum}^{*} \text { : } \\
& P_{E S S}^{*}= \begin{cases}P_{E S S \_m i n} & P_{E S S}^{*} \leq P_{E S S \_m i n} \\
P_{E S S \_m a x} & P_{E S S}^{*} \geq P_{E S S \_m a x} \\
P_{H P P \_m a x}-P_{W P P}^{*} & \\
-P_{S P P}^{*} & \text { o.w. }\end{cases}
\end{aligned}
$$

The active power dispatch references in State 2 are defined as below:

$$
\begin{aligned}
P_{W P P}^{*} & =P_{W P P N}-\alpha\left(P_{H P P}-P_{H P P \_ \text {max }}\right) \\
P_{S P P}^{*} & =P_{S P P N}-(1-\alpha)\left(P_{H P P}-P_{H P P \_ \text {max }}\right) \\
P_{E S S}^{*} & = \begin{cases}P_{E S S \_m i n} & P_{E S S}^{*} \leq P_{E S S \_m i n} \\
P_{E S S \_m a x} & P_{E S S}^{*} \geq P_{E S S \_m a x} \\
P_{H P P \_m a x}-P_{W P P}^{*} & \\
-P_{S P P}^{*} P & \text { o.w. }\end{cases}
\end{aligned}
$$

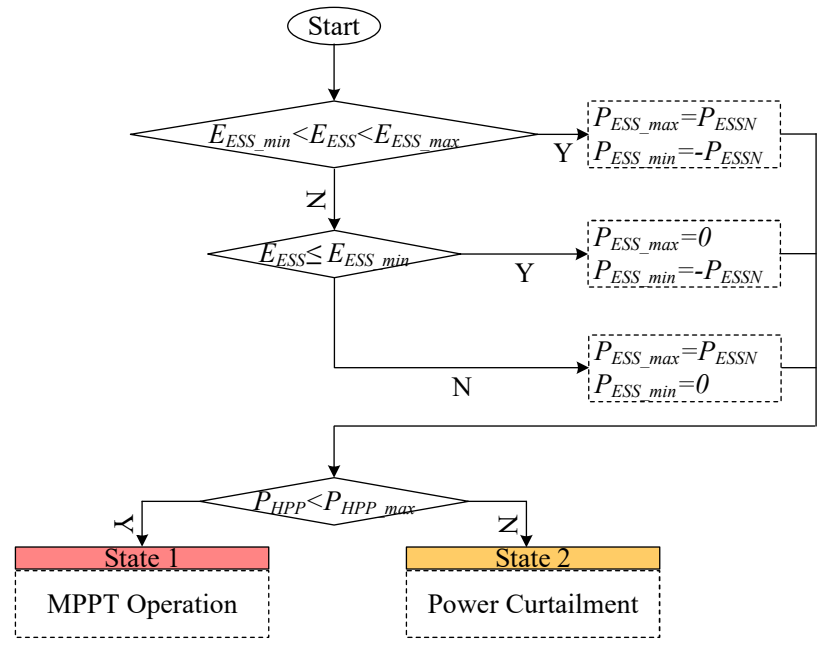

Fig. 5: Control logic for active power dispatch implemented for the HPP dispatch function.

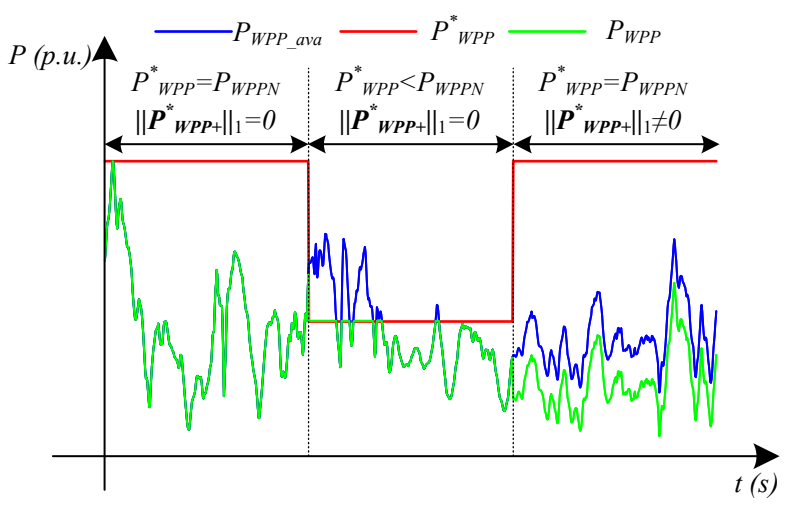

Fig. 6: WPP active power control functions.

where $\alpha$ is the participation factor for WPP power curtailment, and then $1-\alpha$ is the participation factor for SPP power curtailment.

In State 1, active power references for the WPP and the SPP calculated by the HPP dispatch function in (2a) and (2b) are the nominal capacity of the WPP and the SPP instead of available active power from the WPP and the SPP. As long as the renewable power plants receive active power references equal to the nominal capacity, the plant controllers are informed to operate under MPPT mode, as shown in the first section of Fig. 6. As a consequence of the closed-loop control at HPP control level, the ESS performs power reference tracking as (2c) and (2d) as long as its power and energy limits are respected. In State 2, the ESS performs power regulation as (3c) to drive the HPP active power output below $P_{H P P_{-} \max }$. In the meanwhile, active power references for the WPP and the SPP are decreased to reduce their active power output. The plant controllers interpret these active power references that deviate from the nominal capacity as the indicators of the balance control mode, as shown in the second section of Fig. 6. Besides, both states are subject to non-zero active power reserve references, which activate the delta control mode, as shown in the third section of Fig. 6 . 


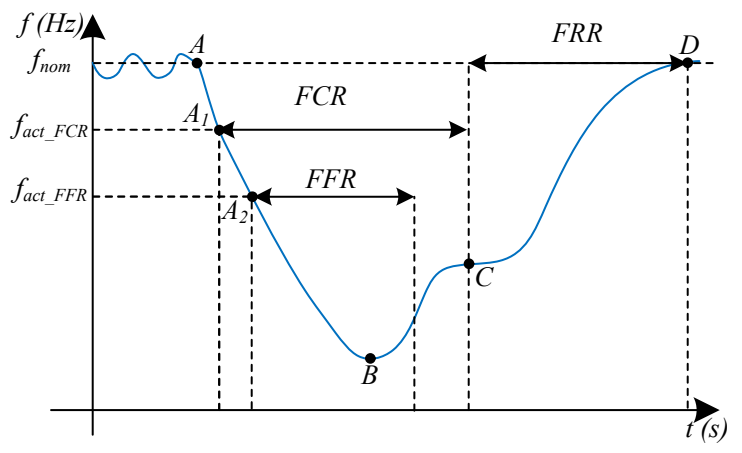

Fig. 7: A typical frequency response in the case of an underfrequency event.

Since both the WPP controller and the SPP controller have immediate access to plant-level available active power, they use this information, along with active power references and active power reserve references sent from the HPP controller, to decide the actual active power output at the plant PoC. Fig. 8 shows a generic plant active power control for WPP and SPP. It is also applicable to the ESS controller by simply dropping the available active power input $P_{p_{-} a v a}$.

The above-mentioned design for active power dispatch has several features that are worth mentioning. First, the proposed active power dispatch in (2a) - (3c) avoids using available active power sent from the WPP controller and the SPP controller. It is based on the consideration that available active power sent from the WPP controller and the SPP controller is delayed in reality due to communication latency, and therefore inaccurate for real-time dispatch at HPP control level. By relying on the anti-windup PI controller to track active power at the HPP PoC rather than using the delayed information, the robustness of the proposed active power dispatch against communication latency is improved. Second, active power references $P_{W P P}^{*}$ and $P_{S P P}^{*}$ sent from the HPP controller are used as more the absolute power limit than the reference, by the WPP controller and the SPP controller. This makes the implementation of both the HPP controller and the plant controller simple and straightforward, and is also aligned with the latest industrial practice.

\section{Frequency Control}

Once the HPP makes a commitment to frequency services in the reserve market, the frequency services are activated when the frequency event happens. A typical frequency response after an under-frequency event is shown in Fig. 7. ENTSOE specifies reserves used for frequency control consisting of FFR, FCR and FRR. The description of FFR, FCR and FRR can be found in [31] and [33].

Since the response time of each frequency service differs, the corresponding control needs to be taken care of at different control levels. In this paper, the control for FFR and FCR is implemented at asset control level, considering the fact that the response time for these two frequency services is short. Since the FRR response is relatively slow, the control for FRR is implemented at HPP control level by adding an extra active

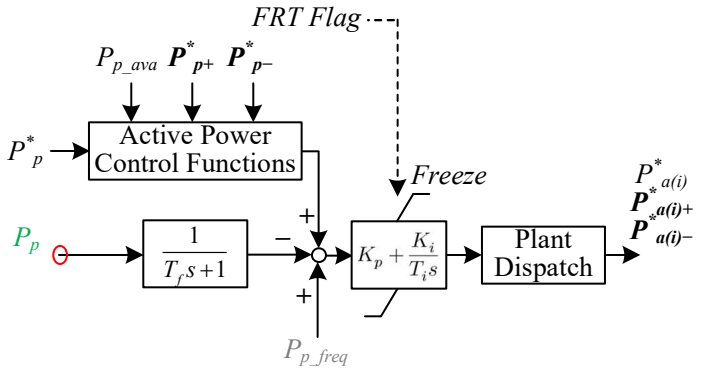

Fig. 8: Plant active power control with FRT freezing mechanism.

power reference $P_{F R R_{-} s e t}$, controlled by the signal directly from system operators, to the HPP active power control. Once activated, the updated active power reference at HPP control level are then dispatched via the active power dispatch algorithm to plant control level via and further down to asset control level, as described in Section III-B.

During the period when asset control level responds to frequency events, both plant and HPP control level should be notified of the action at asset control level in order to avoid counteraction across the control hierarchy. A coordination strategy based on disturbance observer (DOB) is therefore introduced in this paper. The main idea behind this proposed strategy is to consider frequency responses triggered at asset control level as the disturbance to HPP control level and plant control level. As long as the disturbance, which is the combined response of FFR and FCR, is properly estimated by the plant DOB and the HPP DOB, the estimated value is added to the control input as the compensation term to cancel out the disturbance from the measurement. At plant control level, the compensation term from DOB is noted as $P_{p_{-} \text {freq }}$ as shown in Fig. 8, while in HPP control level, the compensation term from DOB is noted as $P_{H P P_{-} f r e q}$ as shown in Fig. 3.

DOB was initially proposed in [34] to improve the robustness of the controllers for DC servo motor systems, given disturbances and uncertainties in the system. As shown in Fig. 9a, the disturbance is written as:

$$
d_{l}(s)=\left[G_{n}(s)^{-1}-G(s)^{-1}\right] y(s)+d(s)+G_{n}(s)^{-1} n(s)
$$

The first item relates to the disturbance introduced by the mismatch between the physical system $G(s)$ and the nominal model $G_{n}(s)$. The second item relates to the external disturbance $d(s)$. The third item relates to the disturbance caused by measurement noise. Therefore, $d_{l}$, also called the lumped disturbance [35], captures all the disturbance and the uncertainties in the system. The filter $Q(s)$ is used to estimate the lumped disturbance, give by:

$$
d_{l_{-} e s t}(s)=Q(s) d_{l}(s)
$$

Note that the DOB shown in Fig. 9a is not the same as the original DOB design [35]. The original DOB design adds the estimated disturbance directly to the output of the controller $C(s)$ to suppress disturbance and uncertainty [35]. Here in the proposed design, the estimated disturbance firstly passes the nominal model $G_{n}(s)$, and the output is then added to 


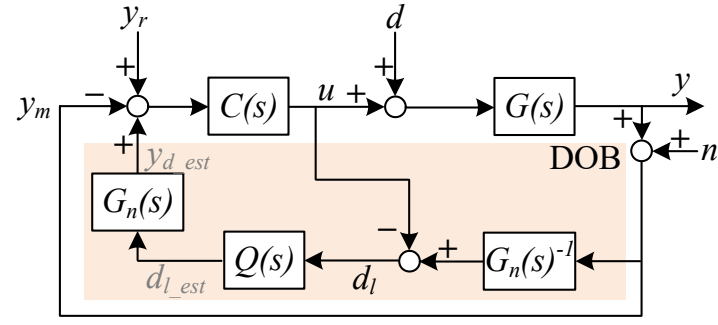

(a) A generic DOB structure

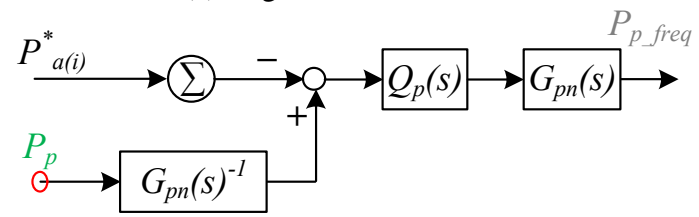

(b) DOB at plant control level

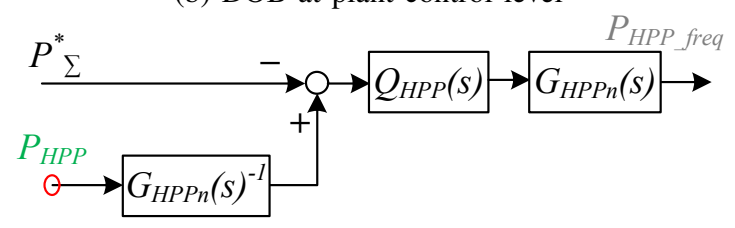

(c) DOB at HPP control level

Fig. 9: DOB-based coordinated frequency control.

the input of the controller $C(s)$ in order to compensate the influence of the disturbance on the measurement. There are differences because the disturbance considered in this paper is supposed not to be suppressed but only to be estimated at plant control level and HPP control level for the sake of coordination. The equivalent control system considering the DOB is expressed as:

$$
u(s)=G_{y_{r} u}(s) y_{r}(s)+G_{d u}(s) d(s)+G_{n u}(s) n(s)
$$

where $G_{y_{r} u}, G_{d u}(s)$ and $G_{n u}(s)$ are the transfer functions from $y_{r}(s)$ to $u(s), d(s)$ to $u(s)$ and $n(s)$ to $u(s)$, respectively. The filter $Q(s)$ and the controller $C(s)$ should be designed in such a way that the controller tracks $y_{r}(s)$ while being insensitive to $d(s)$ and $n(s)$.

Fig. 9b shows the DOB design at plant control level. The active power reference for each unit is summed as one aggregated control output. Active power measurement at the plant $\mathrm{PoC}$ is passed through the inverse of the nominal power plant model $G_{p n}(s)$, from which the aggregated control output is subtracted. The error obtained then passes the plant DOB filter $Q_{p}(s)$. The output of $Q_{p}(s)$ is the estimate of frequency control actions from all the units within the power plant. After it passes the nominal plant model $G_{p n}(s)$, then the estimate of the combined response of FFR and FCR within the power plant is obtained and sent to the plant active power control shown in Fig. 8. The HPP DOB is designed likewise. As shown in Fig. 9c, active power measurement at the HPP PoC is passed through the inverse of the nominal HPP model $G_{H P P n}(s)$. The output of HPP active power control is also used. The output of the HPP DOB filter $Q_{H P P}(s)$ is the estimate of frequency control actions from all the sub-plants. After it passes the nominal HPP model $G_{H P P n}(s)$, the estimate of the combined response of FFR and FCR across the HPP is obtained and then sent to the HPP active power control shown in Fig. 3. Note that each DOB only uses the signals from its own control level, and therefore communication is avoided during frequency service provision.

It is obvious that the design of the filters $Q_{p}(s)$ and $Q_{H P P}(s)$ plays a significant role in the DOB. Design principles for the $Q(s)$ filter can be found in [35], [36]. For example, its relative degree should be higher than that of the nominal plant such that $Q(s) G_{n}(s)^{-1}$ is implementable. The details of DOB design and analysis for hierarchical frequency control will be considered as the next step of this study.

In addition to control coordination, the HPP controller needs to secure the committed reserve as mentioned in Section II-C. The reserve references are sent from the HPP EMS to the HPP controller, and need to be secured by the HPP controller in a real-time fashion. The HPP dispatch function allocates reserve requirements and sends reserve reference to each sub-plant.

Control algorithms for HPP active power reserve dispatch include one for upward reserve dispatch and the other for downward reserve dispatch, both of which are implemented using a priority-list approach. The upward reserve dispatch is executed at first. It firstly allocates the reserve to the sub-plant that has the first priority. If the available power of this subplant, updated by the plant controller, is insufficient to fulfil the reserve reference, then the allocation goes to the plant with the second and the third priority until the reserve requirement is met. After the upward reserve dispatch is completed, the downward reserve dispatch begins. The maximum downward reserves for the WPP and the SPP are calculated initially based on the committed upward reserves. The maximum downward reserve for the ESS is initialized to the maximum charging power. Then, the downward reserve dispatch follows a similar procedure to the upward reserve dispatch. The active power reserve dispatch is updated in a real-time fashion with the available active power updated over time by the plant controllers.

After reserve references are received, plant controllers keep reserves by updating upper and lower power limits in their active power control loops. With respect to WPPs and SPPs, the upper and lower power limits are defined as:

$$
\begin{aligned}
& P_{W P P \_ \text {max }}=P_{W P P \_a v a}-\left\|\mathbf{P}_{\mathbf{W P P}+}^{*}\right\|_{1} \\
& P_{W P P \_ \text {min }}=0 \\
& P_{S P P \_ \text {max }}=P_{S P P \_a v a}-\left\|\mathbf{P}_{\mathbf{S P P}+}^{*}\right\|_{1} \\
& P_{S P P \_ \text {min }}=0
\end{aligned}
$$

If the active power reference received for plant controllers is equal to the nominal capacity of the power plant, the actual active power reference for plant controllers is subject to an upper power limit jointly decided by available active power and active power reserve reference. If active power reserve reference is equal to zero, then the power plant operates under MPPT mode. Otherwise, it operates under the delta control mode. If the active power reference from the HPP controller is less than the nominal capacity, the balance control mode 
is then indicated and the actual active power reference is the minimum of the upper power limit and the active power reference.

In terms of ESSs, the upper and lower power limits are defined as:

$$
\begin{aligned}
& P_{E S S \_ \text {max }}=P_{E S S N}-\left\|\mathbf{P}_{\mathbf{E S S}+}^{*}\right\|_{1} \\
& P_{E S S \_ \text {min }}=-\left(P_{E S S N}-\left\|\mathbf{P}_{\text {ESS }-}^{*}\right\|_{1}\right)
\end{aligned}
$$

By doing so, the ESS secures the reserve for providing frequency service but compromises the capability for regulating HPP active power output. Note that this trade-off is assumed to be handled by the HPP EMS by optimizing the bids between energy markets and ancillary service markets. The HPP controller executes the decision of the HPP EMS at best effort. Through the above-mentioned design, the proposed control architecture enables stacked operation of active power control and frequency control with proper coordination across the control hierarchy.

\section{Reactive Power and Voltage Control}

The main goal of the HPP controller for reactive power and voltage control is to make sure reactive power reference or voltage reference defined by system operator is tracked at the HPP PoC. The output of the anti-windup PI controller is sent as the input to the HPP dispatch function which makes decision on dispatching reactive power reference to each power plant, including auxiliary plants for reactive compensation. The reactive power dispatch algorithm is written as:

$$
\begin{gathered}
Q_{W P P}^{*}=k_{p f_{-} W P P} Q_{\sum}^{*}=\frac{Q_{W P P_{\_} a v a}}{Q_{\sum_{\_} a v a}} Q_{\Sigma}^{*} \\
Q_{S P P}^{*}=k_{p f_{-} S P P} Q_{\sum}^{*}=\frac{Q_{S P P_{\_} a v a}}{Q_{\sum_{\_} a v a}} Q_{\sum}^{*} \\
Q_{E S S}^{*}=k_{p f_{-} E S S} Q_{\sum}^{*}=\frac{Q_{E S S_{-} a v a}}{Q_{\sum \_a v a}} Q_{\sum}^{*} \\
Q_{A U X}^{*}=k_{p f_{-} A U X} Q_{\sum}^{*}=\frac{Q_{A U X_{\_} a v a}}{Q_{\sum_{\_} a v a}} Q_{\sum}^{*}
\end{gathered}
$$

where $k_{p f_{-} W P P}, k_{p f_{-} S P P}, k_{p f_{-} E S S}$ and $k_{p f_{-} A U X}$ are the participation factors for WPP, SPP, ESS and AUX, respectively. $Q_{\sum_{-} a v a}$ is the total of the available reactive power measurements from all the sub-plants, and is received via communication from plant control level.

As shown in Fig. 10, each plant controller regulates the reactive power output at its own plant $\mathrm{PoC}$ based on the reference it receives, and generates either reactive power or delta voltage reference for each asset. These two types of references for asset control level are introduced to accommodate variations of control interfaces from different manufacturers. If the reference is based on reactive power, then it is allocated by the plant dispatch function and dispatched to asset control level, tracked by the asset controller. In the case where the plant controller generates a delta voltage reference, this reference sent to each asset controller is identical in order to guarantee the coordination of plant $\mathrm{PoC}$ voltage regulation among all the units.

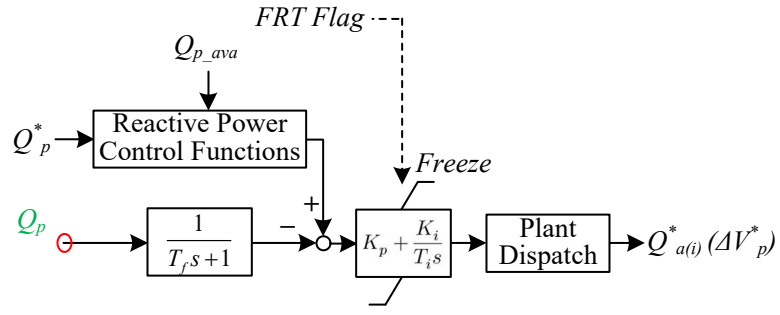

Fig. 10: Plant reactive power control with FRT freezing mechanism.

\section{E. Fault Ride-Through}

In the case of FRT, the asset control injects reactive currents with respect to the grid code [37] while avoids overloading the inverter and prevents overcharging the DC-link capacitor in power converters. Although the control action mainly happens at asset control level, coordination is needed for HPP control level and plant control level to avoid counteracting with local control actions.

As shown in Figs. 8 and 10, a freezing mechanism is implemented for FRT for both active power control and reactive power control at plant control level. While the freezing of the active power control is intended for avoiding error accumulation resulted from an immediate decrease in active power, the freezing of the reactive power control avoids plant controllers from counteracting with an instant injection of reactive current. The freezing signal becomes active when FRT events are detected. Note that only is the local voltage measurement used, such that no communication is required during the FRT period. The inputs of the PI controllers are held at zero during the freezing period, and therefore the outputs of the controllers are held at the pre-fault value. The same mechanism for FRT is also applied to HPP controllers as shown in Fig. 3 for the same reason.

\section{CONCLUSION}

This paper proposes an innovative hierarchical control architecture for co-located HPPs, where four control levels are included, with information exchange across the control hierarchy being discussed in detail. A general framework for control functions, including active power control, frequency control, reactive power control and voltage control, is developed as well, with the consideration that all the control levels act in coordination with each other. The aspect of communication latency and stacked operation is also accounted for in the proposed architecture. Detailed design for each control function will be included as future work.

\section{ACKNOWLEDGMENT}

The work is done as a part of Indo-Danish HYBRIDize project funded by Innovation Fund Denmark (IFD) with grant number 8127-00015B. 


\section{REFERENCES}

[1] W. Gorman, A. Mills, M. Bolinger, R. Wiser, N. G. Singhal, E. Ela, and E. O'Shaughnessy, "Motivations and options for deploying hybrid generator-plus-battery projects within the bulk power system," Electricity Journal, vol. 33, no. 5, p. 106739, 2020. [Online]. Available: https://doi.org/10.1016/j.tej.2020.106739

[2] WindEurope, "Renewable hybrid power plants - WindEurope." [Online]. Available: https://windeurope.org/intelligenceplatform/product/renewable-hybrid-power-plants-interactive-tool/

[3] Q. Guo, H. Sun, B. Wang, B. Zhang, W. Wu, and L. Tang, "Hierarchical automatic voltage control for integration of large-scale wind power: Design and implementation," Electric Power Systems Research, vol. 120, 2015.

[4] S. Corsi, M. Pozzi, C. Sabelli, and A. Serrani, "The coordinated automatic voltage control of the Italian transmission grid - Part I: Reasons of the choice and overview of the consolidated hierarchical system," IEEE Transactions on Power Systems, vol. 19, no. 4, 2004.

[5] J. M. Guerrero, J. C. Vasquez, J. Matas, L. G. De Vicuña, and M. Castilla, "Hierarchical control of droop-controlled AC and DC microgrids - A general approach toward standardization," IEEE Transactions on Industrial Electronics, vol. 58, no. 1, pp. 158-172, 2011.

[6] R. Teodorescu, M. Liserre, and P. Rodríguez, Grid converters for photovoltaic and wind power systems, 2010.

[7] V. Gevorgian and B. O'Neill, "Advanced grid-friendly controls demonstration project for utility-scale PV power plants," National Renewable Energy Laboratory, Golden, CO, USA, Tech. Rep. NREL/TP-5D00-65368, 2016. [Online]. Available: https://www.nrel.gov/docs/fy16osti/65368.pdf

[8] C. Loutan, P. Klauer, S. Chowdhury, S. Hall, M. Mrjaria, V. Chadliev, N. Milam, C. Milan, and V. Gevorgian, "Demonstration of essential reliability services by a 300-MW solar photovoltaic power plant," National Renewable Energy Laboratory, Golden, CO, USA, Tech. Rep. NREL/TP-5D00-67799, 2017.

[9] J. R. Kristoffersen and P. Christiansen, "Horns Rev Offshore Windfarm: Its main controller and remote control system," Wind Engineering, vol. 27, no. 5, pp. 351-360, 2003.

[10] E. Ela, V. Gevorgian, P. A. Fleming, Y. C. Zhang, M. Singh, E. Muljadi, A. Scholbrook, J. Aho, A. Buckspan, L. Y. Pao, V. Singhvi, A. Tuohy, P. Pourbeik, D. Brooks, and N. Bhatt, "Active power controls from wind power: bridging the gaps," National Renewable Energy Laboratory, Golden, CO, USA, Tech. Rep. NREL/TP-5D00-60574, 2014. [Online]. Available: http://www.nrel.gov/docs/fy14osti/60574.pdf

[11] A. Nagarajan, D. Cutler, A. Latif, X. Li, R. Bryce, Y. Shi, J. Tan, Q. Long, P. Gotseff, E. Du, Y. Hirata, K. Yamanishi, K. Yano, R. Kitano, Y. Nagaoka, A. Nagarajan, D. Cutler, A. Latif, X. Li, R. Bryce, Y. Shi, J. Tan, Q. Long, P. Gotseff, and E. Du, "Value streams from distribution grid support using utility-scale vanadium redox flow battery value streams from distribution grid support using utility-scale vanadium redox flow battery demonstration project," National Renewable Energy Laboratory, Golden, CO, USA, Tech. Rep. NREL/TP-5D00-71545, 2018.

[12] A. D. Hansen, P. Sørensen, F. Iov, and F. Blaabjerg, "Centralised power control of wind farm with doubly fed induction generators," Renewable Energy, vol. 31, no. 7, pp. 935-951, 2006.

[13] A. F. Hoke, M. Shirazi, S. Chakraborty, E. Muljadi, and D. Maksimovic, "Rapid active power control of photovoltaic systems for grid frequency support," IEEE Journal of Emerging and Selected Topics in Power Electronics, vol. 5, no. 3, pp. 1154-1163, 2017.

[14] I. D. Margaris, S. A. Papathanassiou, N. D. Hatziargyriou, A. D. Hansen, and P. Sorensen, "Frequency control in autonomous power systems with high wind power penetration," IEEE Transactions on Sustainable Energy, vol. 3, no. 2, pp. 189-199, 2012.

[15] J. Licari, J. Ekanayake, and I. Moore, "Inertia response from full-power converter-based permanent magnet wind generators," Journal of Modern Power Systems and Clean Energy, vol. 1, no. 1, pp. 26-33, 2013.

[16] H. Wang, Z. Chen, and Q. Jiang, "Control method for variable speed wind turbines to support temporary primary frequency control," Proceedings of the IEEE Power Engineering Society Transmission and Distribution Conference, no. 1, pp. 1-5, 2014.

[17] Z. Wu, W. Gao, T. Gao, W. Yan, H. Zhang, S. Yan, and X. Wang, "Stateof-the-art review on frequency response of wind power plants in power systems," Journal of Modern Power Systems and Clean Energy, vol. 6, no. 1, pp. 1-16, 2018.

[18] B. R. Karthikeya and R. J. Schutt, "Overview of wind park control strategies," IEEE Transactions on Sustainable Energy, vol. 5, no. 2, pp. 416-422, 2014.
[19] R. K. Varma and M. Akbari, "Simultaneous fast frequency control and power oscillation damping by utilizing PV solar system as PVSTATCOM," IEEE Transactions on Sustainable Energy, vol. 11, no. 1, pp. 415-425, 2020.

[20] Q. Li and M. E. Baran, "A novel frequency support control method for PV plants using tracking LQR," IEEE Transactions on Sustainable Energy, vol. 11, no. 4, pp. 2263-2273, 2020.

[21] S. I. Nanou, A. G. Papakonstantinou, and S. A. Papathanassiou, "A generic model of two-stage grid-connected PV systems with primary frequency response and inertia emulation," Electric Power Systems Research, vol. 127, pp. 186-196, 2015. [Online]. Available: http://dx.doi.org/10.1016/j.epsr.2015.06.011

[22] H. Xin, Y. Liu, Z. Wang, D. Gan, and T. Yang, "A new frequency regulation strategy for photovoltaic systems without energy storage," IEEE Transactions on Sustainable Energy, vol. 4, no. 4, pp. 985-993, 2013.

[23] Y. Liu, J. R. Gracia, T. J. King, and Y. Liu, "Frequency regulation and oscillation damping contributions of variable-speed wind generators in the U.S. Eastern Interconnection (EI)," IEEE Transactions on Sustainable Energy, vol. 6, no. 3, pp. 951-958, 2015.

[24] H. Karbouj and Z. H. Rather, "Voltage control ancillary service from wind power plant," IEEE Transactions on Sustainable Energy, vol. 10, no. 2, pp. 759-767, 2019.

[25] A. D. Hansen and G. Michalke, "Multi-pole permanent magnet synchronous generator wind turbines' grid support capability in uninterrupted operation during grid faults," IET Renewable Power Generation, vol. 3, no. 3, pp. 333-348, 2009.

[26] G. Lammert, D. Premm, L. D. P. Ospina, J. C. Boemer, M. Braun, and T. Van Cutsem, "Control of photovoltaic systems for enhanced short-term voltage stability and recovery," IEEE Transactions on Energy Conversion, vol. 34, no. 1, pp. 243-254, 2019.

[27] R. K. Varma and S. Mohan, "Mitigation of fault induced delayed voltage recovery (FIDVR) by PV-STATCOM," IEEE Transactions on Power Systems, vol. 35, no. 6, pp. 4251-4262, 2020.

[28] J. Kim, E. Muljadi, J. W. Park, and Y. C. Kang, "Adaptive hierarchical voltage control of a DFIG-based wind power plant for a grid fault," IEEE Transactions on Smart Grid, vol. 7, no. 6, pp. 2980-2990, 2016.

[29] X. Li, D. Hui, and X. Lai, "Battery energy storage station (BESS)-based smoothing control of photovoltaic (PV) and wind power generation fluctuations," IEEE Transactions on Sustainable Energy, vol. 4, no. 2, pp. 464-473, 2013

[30] E. Bullich-Massagué, M. Araguiés-Peñalba, A. Sumper, and O. BoixAragones, "Active power control in a hybrid PV-storage power plant for frequency support," Solar Energy, vol. 144, no. 2015, pp. 49-62, 2017.

[31] D. V. Pombo, F. Iov, and D. I. Stroe, "A novel control architecture for hybrid power plants to provide coordinated frequency reserves," Energies, vol. 12, no. 5, 2019.

[32] F. Calero, C. A. Canizares, and K. Bhattacharya, "Dynamic modeling of battery energy storage and applications in transmission systems," IEEE Transactions on Smart Grid, vol. 12, no. 1, pp. 589-598, 2021.

[33] R. Eriksson, N. Modig, and M. Kuivaniemi, "Ensuring future frequency stability in the Nordic Synchronous Area," in 18th International Wind Integration Workshop, Dublin, Ireland, 2019, pp. 1-7.

[34] K. Ohishi, M. Nakao, K. Ohnishi, and K. Miyachi, "Microprocessorcontrolled DC motor for load-insensitive position servo system," IEEE Transactions on Industrial Electronics, vol. IE-34, no. 1, 1987.

[35] W. H. Chen, J. Yang, L. Guo, and S. Li, "Disturbance-Observer-Based Control and Related Methods - An Overview," IEEE Transactions on Industrial Electronics, vol. 63, no. 2, pp. 1083-1095, 2016.

[36] T. Umeno, T. Kaneko, and Y. Hori, "Robust servosystem design with two degrees of freedom and its application to novel motion control of robot manipulators," IEEE Transactions on Industrial Electronics, vol. 40, no. 5, 1993.

[37] Y. Yang, F. Blaabjerg, and H. Wang, "Low-voltage ride-through of single-phase transformerless photovoltaic inverters," IEEE Transactions on Industry Applications, vol. 50, no. 3, pp. 1942-1952, 2014. 